\title{
Orchidaceae na região central de São Paulo, Brasil
}

\author{
Orchidaceae in the central portion of São Paulo state, Brazil
}

\author{
Alessandro Wagner Coelho Ferreira ${ }^{1}$, \\ Maria Inês Salgueiro Lima ${ }^{2} \&$ Emerson Ricardo Pansarin ${ }^{3}$
}

\begin{abstract}
Resumo
Este estudo apresenta o levantamento florístico de Orchidaceae ocorrentes em 125 fragmentos florestais de 23 municípios da região central do estado de São Paulo. Foram identificadas 218 espécies distribuídas em 96 gêneros. Os indivíduos coletados em estado vegetativo foram mantidos em casa de vegetação até a ocorrência de floração. A maioria das espécies (64\%) é epífita, 35\% são terrícolas, $14,6 \%$ são rupícolas, duas são hemiepífitas $(0,9 \%)$ e apenas uma é micoheterotrófica $(0,4 \%)$. Os gêneros mais representativos são Acianthera (13 spp.), Habenaria e Gomesa (12 spp.) e Epidendrum (11 spp.). Dentre as espécies não identificadas, quatro parecem ser novas para a ciência: Anathallis sp., Baskervilla sp., Pelexia sp., Triphora sp. Além disso, uma nova combinação para o gênero Gomesa $\mathrm{R}$.Br. é apresentada. O elevado número de espécies encontradas provavelmente está relacionado ao fato da região ser um ecótono entre o Cerrado e a Floresta Atlântica. Muitas das espécies $(44,7 \%)$ são raras na região e encontram-se ameaçadas devido ao intenso desmatamento e coletas indiscriminadas.

Palavras-chave: Cerrado, ecótono, Floresta Atlântica, levantamento florístico.
\end{abstract}

\begin{abstract}
This study reports the floristic survey of Orchidaceae occurring in 125 forest fragments distributed among 23 counties of the central region of São Paulo state, Brazil. In this region, the family is characterized by 219 species distributed in 96 genera. Individuals collected in the vegetative state were maintained in a greenhouse until flowering. The majority of species (64\%) are epiphytes, while $35 \%$ are terrestrial, $14.6 \%$ are rupicolous, two $(0.9 \%)$ are hemi-epiphytes and one is mycoheterotrophic (0.4\%). Acianthera (13 spp.), Habenaria and Gomesa (12 spp.), and Epidendrum (11 spp.) are the most representative genera. Of the unidentified species, four are possibly new to science: Anathallis sp., Baskervilla sp., Pelexia sp., Triphora sp. Furthermore, a new combination for the genus Gomesa R.Br. is presented. The central portion of São Paulo state is an ecotone between Brazilian Savanna and Atlantic Forest with a high number of species from both formations. Many species $(44.7 \%)$ are rare in the study area and are threatened due to anthropogenic disturbance and indiscriminate collecting of orchids.
\end{abstract}

Key words: Savanna, ecotone, Atlantic Forest, floristic survey.

\section{Introdução}

Orchidaceae é cosmopolita em distribuição e estima-se que possua 24.500 espécies (Dressler 2005). De acordo com Pabst \& Dungs (1975), o Brasil possui aproximadamente 2.300 espécies e 191 gêneros. As espécies de orquídeas podem ser terrícolas, palustres, epífitas e rupícolas. No entanto, a grande maioria delas ocorre como epífita. Em média, duas em cada três espécies de Orchidaceae são encontradas sobre árvores (e.g., Dressler 1981, 1993). Segundo Kersten (2006), devido à dependência da umidade e do substrato arbóreo, a diversidade de epífitas é um indicador ecológico da qualidade ambiental.

${ }^{1}$ Doutorando do Programa de Pós-Graduação em Ecologia e Recursos Naturais da Universidade Federal de São Carlos, Depto. Botânica, Rod. Washington Luis km 235, C.P. 676, 13565-905, São Carlos, SP. alessandrowcf@yahoo.com.br

${ }^{2}$ Universidade Federal de São Carlos, Depto. Botânica, Rod. Washington Luis km 235, C.P. 676, 13565-905, São Carlos, SP. ines@ufscar.br

${ }^{3}$ Autor para correspondência: Universidade de São Paulo, Faculdade de Filosofia, Ciências e Letras de Ribeirão Preto, Depto. Biologia, 14040-901, Ribeirão Preto, SP. epansarin@ffclrp.usp.br 
Segundo Hoehne (1949), espécies de Orchidaceae podem ser encontradas em todos os tipos de vegetação no Brasil, sendo as regiões de Floresta Atlântica e de Cerrado as mais ricas em diversidade (e.g., Batista \& Bianchetti 2003; Romanini \& Barros 2007).

A porção central do estado de São Paulo é caracterizada como um ecótono entre os dois biomas "hotspots" brasileiros: Cerrado sensu lato e Floresta Atlântica Mesófila Estacional Semidecidual (Kronka et al. 1993; Myers et al. 2000). A vegetação nativa dessa região vem sendo fragmentada ao longo dos anos devido à intensa atividade econômica. Estimase que cerca de $96 \%$ do cerrado já foi derrubado, principalmente a partir de 1960 (Kronka et al. 1998; Soares et al. 2003). Além do Cerrado, as Florestas Mesófilas Estacionais Semideciduais também vêm sendo devastadas principalmente pelo avanço das lavouras de cana-de-açúcar. Apesar dessa degradação, poucos estudos de florística de Orchidaceae têm sido realizados abrangendo esses tipos de vegetação no Estado (para exceções veja Cardoso \& Israel 2005 e Pansarin \& Pansarin 2008). Em São Paulo estudos florísticos envolvendo Orchidaceae concentram-se em áreas de Florestas Ombrófilas, em regiões litorâneas (Barros 1983; Ribeiro 1992; Romanini \& Barros 2007). Tal constatação reforça a necessidade de se intensificar o conhecimento florístico da família para outras formações, uma vez que o conhecimento da diversidade das espécies é um dos fatores que subsidiam a preservação dos ecossistemas (e.g., Pansarin \& Pansarin 2008).

Baseando-se nesses fatos o presente trabalho teve como objetivo realizar o inventário florístico das Orchidaceae da região central do estado de São Paulo. Além disso, foram obtidos dados sobre os períodos de floração, hábitos, habitats e municípios de ocorrência. Em virtude das recentes alterações nomenclaturais realizadas por Chase et al. (2009) não contemplarem todas as espécies classicamente consideradas como Oncidium encontradas na região de estudo, o presente trabalho traz a trasferência de O. fimbriatum para Gomesa R.Br.

\section{Material e Métodos Local de estudo}

Foram visitados 125 fragmentos florestais distribuídos entre 23 municípios da região central do estado de São Paulo, como segue: Analândia, Araraquara, Boa Esperança do Sul, Brotas, Charqueada, Corumbataí, Descalvado, Dourado,
Gavião Peixoto, Guatapará, Ibaté, Ipeúna, Itirapina, Jaboticabal, Luís Antônio, Matão, Monte Alto, Ribeirão Bonito, Rincão, São Carlos, São Pedro, Torrinha e Trabijú. A área destes fragmentos variou desde um até cerca de 150 hectares. Eles estão em uma região de transição entre os domínios do Cerrado e da Floresta Atlântica (Kronka et al. 1993; Myers et al. 2000). Os fragmentos ficam inseridos em um polígono irregular com aproximadamente $4500 \mathrm{~km}^{2}$ (Fig. 1).

Tal polígono é delimitado por oito vértices demarcados pelas seguintes coordenadas geográficas: 1- Monte Alto: $21^{\circ} 14{ }^{\prime} 55^{\prime} ' S$ e 48 29'06”O; 2- Jaboticabal: $21^{\circ} 14 ' 53$ ”S e 48 16'03”O; 3- Luís Antônio (Estação Ecológica de Jataí): 21 33'05'S e 4743'11'O; 4- Corumbataí: 22 '13'58'S e 47³5'51'O; 5- São Pedro: 22³2'15'’S e 4755'06'O; 6- Torrinha: 22 23'47'S e 48 13'58'O; 7- Boa Esperança do Sul (Fazenda Java): 21 '51'34'’S e 48 21'42'OO e, 8- Matão: $21^{\circ} 37^{\prime} 11^{\prime \prime}$ S e 48 32'40'O (Fig. 1). A altitude varia entre $550 \mathrm{~m}$ (na depressão periférica, região de Charqueada) e por volta de $1000 \mathrm{~m}$ (nas cuestas basálticas da Serra Geral, entre São Carlos e Analândia). O clima predominante é o temperado úmido, com inverno frio e seco e verão quente e úmido (Cwa, Köppen 1948).

\section{Trabalho de campo e laboratório}

Os fragmentos florestais da região central do estado de São Paulo foram visitados a cada dois meses, desde janeiro de 1996. Porém, entre janeiro de 2006 e fevereiro de 2009, as visitas foram intensificadas e as coletas passaram a ser semanais. Durante a realização do trabalho foram realizadas 425 visitas ao campo. Ao menos três fragmentos florestais foram amostrados por município.

Nestes fragmentos florestais, foram estudadas espécies de orquídeas de três tipos de vegetação: Cerrado sensu lato, incluindo o cerrado sensu stricto, o campo limpo seco e úmido, o campo cerrado-seco e úmido e o cerradão (Coutinho 1978); florestas ripícolas (incluindo matas ciliares, matas galeria, matas de brejo e matas de cachoeiras e corredeiras - segundo Durigan et al. 2004, adaptado); florestas mesófilas estacionais semideciduais que receberam segundo Ramos et al. (2007) diferentes denominações por diferentes autores, como floresta latifoliada da bacia do Paraná-Uruguai, mata atlântica de interior, floresta latifoliada semicaducifólia, floresta tropical latifoliada mesofítica perenifólia de terra firme, floresta mesófila semidecídua e floresta estacional semidecidual. Segundo esses mesmos autores, esta vegetação, juntamente com 

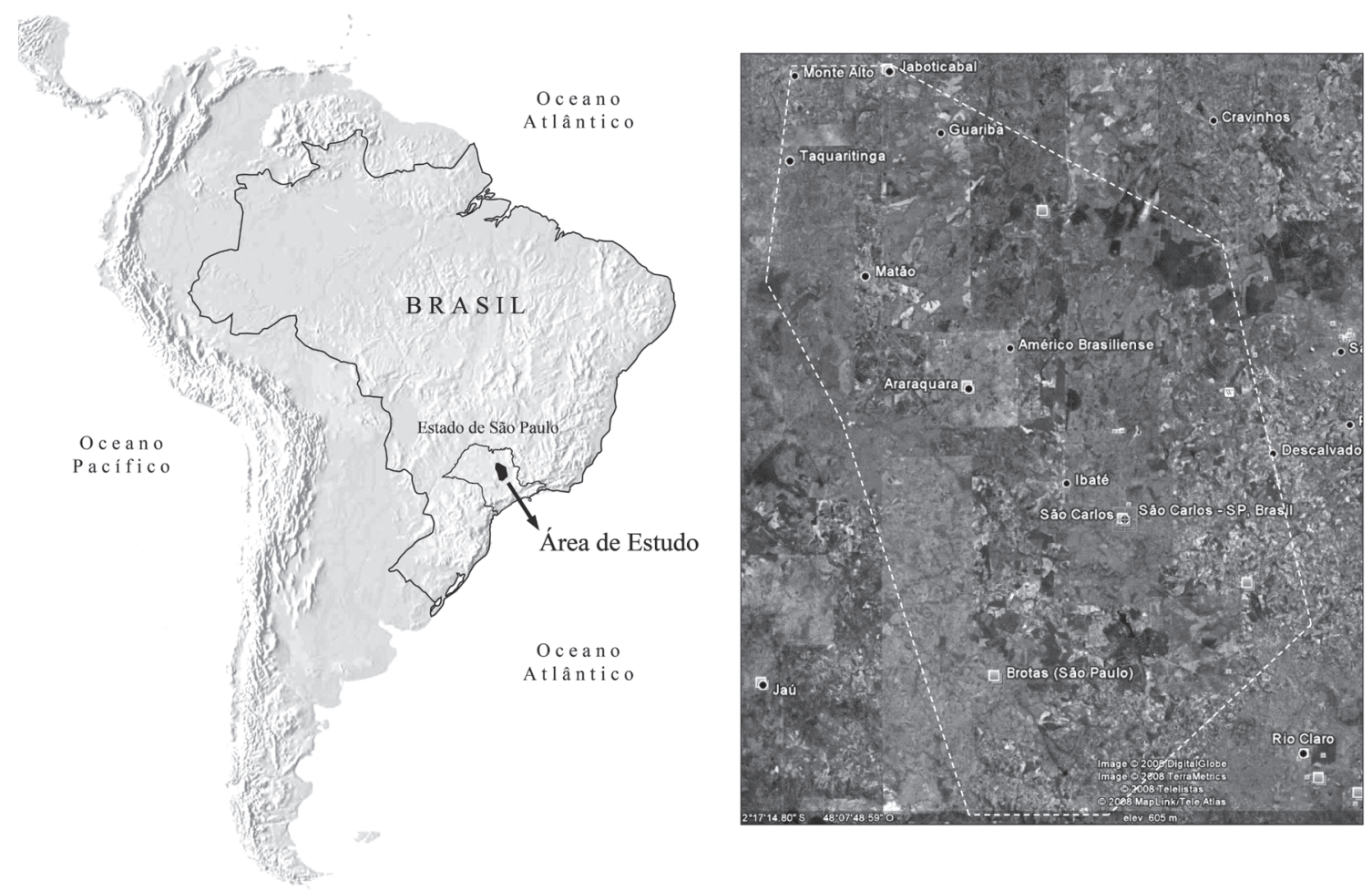

Figura 1 - Localização dos 40 principais pontos de coleta distribuídos entre os 23 municípios visitados do estado de São Paulo, Brasil. Figure 1 - Location of the main 40 points of collections distributed among 23 municipalities visited in São Paulo state, Brazil.

a floresta ombrófila densa (da encosta atlântica) e a floresta ombrófila mista (mata de araucária), compõem o denominado domínio da floresta atlântica, a qual (no território nacional) tem sua área delimitada e protegida pelo Decreto 750, de 10 de fevereiro de 1993, que proíbe o corte, a exploração e a supressão mesma.

A coleta de dados foi aleatória, ou seja, em cada visita os fragmentos foram percorridos sem demarcação de parcelas. Para todas as espécies coletadas foram registradas informações sobre período de floração, hábitos, habitats, bem como os municípios de ocorrência.

Espécies em estado vegetativo foram coletadas no campo e, posteriormente, cultivadas em casa de vegetação na UFSCar, campus São Carlos, até as florações. Todas as espécies foram fotografadas com câmera digital e também sob lupa estereoscópica acoplada ao microcomputador, sendo as imagens obtidas trabalhadas pelo programa Image Pro Plus versão 4.0 (Media Cybernetics, Silver Spring, USA) e utilizadas para auxiliar nas identificações.

Os espécimes férteis, coletados durante as expedições a campo ou que floresceram em cultivo foram herborizados e depositados nos herbários HUFSCar da Universidade Federal de São Carlos, campus São Carlos, SPFR da Universidade de São Paulo, campus Ribeirão Preto e UEC da Universidade Estadual de Campinas.

\section{Identificação das espécies}

Para a identificação das espécies foram utilizadas as principais obras de referência na taxonomia das Orchidaceae brasileiras (Cogniaux 1893-1896, 1898-1902, 1904-1906; Hoehne 1940, 1942 , 1945, 1949, 1953; Pabst \& Dungs 1975, 1977; Sprunger et al. 1996). Também, alguns trabalhos mais específicos de Orchidaceae e obras originais foram consultados (e.g., Pabst 1950; Garay 1977; Luer 1986a,b,c; Toscano de Brito \& Cribb 2005; Romanini \& Barros 2007). A divisão em subfamílias foi apresentada de acordo com Chase et al. (2003). A determinação das autoridades taxonômicas foi realizada de acordo com Kew Monocot (World Checklist of Selected Plant Families 2006).

As espécies foram consideradas como raras quando foram encontrados de um a poucos indivíduos isolados, e em apenas um dos fragmentos visitados. 


\section{Resultados}

Na região central de do estado de São Paulo foram registradas 218 espécies de Orchidaceae distribuídas em 96 gêneros e quatro subfamílias: Epidendroideae, Orchidoideae, Vanilloideae e Cypripedioideae (Tab. 1). Dessas, $64 \%$ são epífitas (140 spp.), 35\% terrícolas (77 spp.), 15,5\% rupícolas (34 spp.), 0,9\% hemiepífitas (duas espécies) e 0,4\% terrícola micoheterotrófica (uma espécie). Todas as espécies encontradas como rupícolas, eventualmente também podem ser encontradas sobre árvores em algumas das áreas aqui investigadas. Os gêneros com maior riqueza de espécies foram: Acianthera (13 spp.), Habenaria e Gomesa (12 spp.) e Epidendrum (11 spp.). Das espécies que não puderam ser identificadas (Tab. 1), quatro (Anathallis sp., Baskervilla sp., Pelexia sp., Triphora sp.) parecem ser novas para a ciência. O gênero Triphora é referido pela primeira vez para o estado de São Paulo. Dentre as espécies encontradas durante o desenvolvimento do presente estudo, $44,7 \%$ são consideradas como raras na região de estudo, sendo encontrado de um a poucos indivíduos isolados.

O habitat com maior número de ocorrências de espécies na região central de São Paulo foi constatado para a floresta ripícola (195 spp.; 89\%), seguido pelas florestas mesófilas estacionais semideciduais (79 spp.; 36\%). No campo cerrado foram encontradas 21 espécies $(9,6 \%)$, enquanto no campo limpo foram registradas $20 \mathrm{spp} .(9,1 \%) \mathrm{e}$ no cerrado sensu stricto e cerradão $18 \mathrm{spp} .(8,2 \%)$. Algumas espécies, como Oeceoclades maculata (Lindl.) Lindl., podem ser encontradas em dois ou mais habitats (Tab. 1).

A maioria das espécies catalogadas (139 spp., $63,4 \%$ ) floresce no outono, entre os meses de março e maio. No verão (entre dezembro e fevereiro) foram registradas 136 espécies $(62,1 \%)$, enquanto que na primavera foram observadas $103 \mathrm{spp}$. (47\%) em floração. O inverno, estação mais seca do ano na região sudeste, é a estação que apresenta o menor número de espécies em floração (Tab. 1).

Como as recentes alterações nomenclaturais incluem todas as espécies classicamente consideradas como Oncidium encontradas na região de estudo, o persente trabalho traz a trasferência de Oncidium fimbriatum para o gênero Gomesa R.Br., como segue:

Gomesa fimbriata (Lindl.) A.C. Ferreira, M.I.S. Lima \& Pansarin, comb. nov.; basiônimo: Oncidium fimbriatum Lindl., Gen. Sp. Orchid. Pl. 199. 1833.

\section{Discussão}

Alguns dos gêneros com maior número de espécies na região de estudo, como Acianthera, Habenaria, Epidendrum são também os mais representativos em levantamentos realizados em outras áreas do estado de São Paulo (Romanini \& Barros 2007; Pansarin \& Pansarin 2008). Acianthera, assim como Campylocentrum, Cyclopogon, Cattleya e Encyclia são comuns em ambientes de floresta atlântica, sendo muito diversos em áreas de floresta ombrófila densa (Barros 1983; Miller et al. 1996; Romanini \& Barros 2007). Já os gêneros Cyrtopodium, Habenaria e Pelexia, encontrados em algumas das áreas abrangidas pelo presente estudo, são comuns em ambientes de Cerrado (Batista \& Bianchetti 2003; Batista et al. 2005). Assim, a existência de considerável número de espécies comuns em ambas formações na porção central do estado de São Paulo pode estar relacionada com o fato de a região ser ecotonal, apresentando elementos dos biomas da Floresta Atlântica e do Cerrado. Alguns gêneros com considerável número de espécies na região de estudo, como Bulbophyllum e Epidendrum, por exemplo, são muito comuns em regiões ecotonais (Menini-Neto et al. 2007; Pansarin \& Pansarin 2008).

Quando comparados os principais levantamentos florísticos realizados no Brasil observa-se que áreas de Floresta Atlântica são as que apresentam o maior número de espécies (Pabst \& Dungs 1975). As áreas ecotonais entre a Floresta Atlântica e o Cerrado apresentam uma menor riqueza de espécies quando comparadas a regiões compostas exclusivamente por Mata Atlântica sensu stricto (i.e., floresta ombrófila densa) (e.g., Romanini \& Barros 2007). Essas áreas ecotonais, no entanto, tendem a ser mais diversas que regiões de Cerrado. O bioma do Cerrado é uma área de convergência de muitas fitofisionomias e pode ocorrer em áreas acidentadas e possuir florestas galeria, intensificando a ocorrência de microhabitats que favorecem a coexistência de diferentes espécies de Orchidaceae (Pabst \& Dungs 1975). O bioma da Floresta Amazônica é o que apresenta menor número de espécies, embora deva-se considerar que áreas desse bioma são pouco representadas em levantamentos florísticos envolvendo a família Orchidaceae (Braga 1977; Silveira et al. 1995; Atzinger et al. 1996; Ribeiro et al. 1999). A homogeneidade da paisagem e a extensa planície parecem não criar muitos microhabitats; apenas nos ambientes amazônicos que apresentam desníveis ou heterogeneidade de fitofisionomias, é que surge uma maior riqueza de espécies de Orchidaceae (Pabst \& Dungs 1975; Braga 1977; Dressler 1981, 1993). 
T̋ Tabela $1-$ Espécies de Orchidaceae encontradas na região central do estado de São Paulo. Hábito: $\mathrm{E}=$ epífita, $\mathrm{HE}=$ hemiepífita, $\mathrm{R}=$ rupícola, $\mathrm{T}=$ terrícola, $\mathrm{TM}=$ terrícola micoheterotrófica em fase adulta. Habitat: $\mathrm{CR}=$ Cerrado sensu stricto, $\mathrm{CCR}=\mathrm{Campo}$ Cerrado, $\mathrm{CLE}=\mathrm{Campo}$ Limpo Estacional, $\mathrm{CRD}=\mathrm{Cerradão}, \mathrm{FES}=\mathrm{Floresta}$ Atlântica Mesófila Estacional Semidecidual, $\mathrm{FR}=$ Floresta Ripícola. Municípios: $\mathrm{AN}=$ Analândia, $\mathrm{AR}=$ Araraquara, $\mathrm{BE}=\mathrm{Boa}$ Esperança do $\mathrm{Sul}$, $\mathrm{BR}=\mathrm{Brotas}, \mathrm{CH}=\mathrm{Charqueada}$ $\mathrm{CO}=$ Corumbataí, $\mathrm{DE}=$ Descalvado, $\mathrm{DO}=$ Dourado, $\mathrm{GP}=$ Gavião Peixoto, $\mathrm{GU}=$ Guatapará, IB = Ibaté, IP = Ipeúna, IT = Itirapina, JB = Jaboticabal, LA = Luís Antônio, $\mathrm{MA}=$ Monte Alto, $\mathrm{MT}=$ Matão, $\mathrm{RB}=$ Ribeirão Bonito, $\mathrm{RI}=$ Rincão, $\mathrm{SC}=$ São Carlos, $\mathrm{SP}=$ São Pedro, $\mathrm{TO}=$ Torrinha, $\mathrm{TR}=\mathrm{Trabijú,} \mathrm{T}=\mathrm{ocorre}$ em todos os municípios Floração: JAN = janeiro, FEV = fevereiro, MAR $=$ março, $\mathrm{ABR}=$ abril, MAI $=$ maio, JUN $=$ junho, JUL $=$ julho, AGO $=$ agosto, $\mathrm{SET}=$ setembro, OUT $=$ outubro, $\mathrm{NOV}$ $=$ novembro, $\mathrm{DEZ}=$ dezembro

Table 1 - Species of orchids occurring at central region of São Paulo State, Brazil. Habit: $\mathrm{E}=$ epiphyte, $\mathrm{HE}=$ hemiepífita, $\mathrm{R}=$ rupiculous, $\mathrm{T}=$ terrestrial, $\mathrm{TM}=$ myco-heterotrophic. Habitat: $\mathrm{CR}$ $=$ Cerrado sensu stricto, $\mathrm{CCR}=$ Campo Cerrado, $\mathrm{CLE}=$ Campo Limpo Estacional, $\mathrm{CRD}=$ Cerradão, FES $=$ Floresta Atlântica Mesófila Estacional Semidecidual, FR $=$ Floresta Ripícola Municipalities: $\mathrm{AN}=$ Analândia, $\mathrm{AR}=$ Araraquara, $\mathrm{BE}=$ Boa Esperança do Sul, $\mathrm{BR}=$ Brotas, $\mathrm{CH}=$ Charqueada, $\mathrm{CO}=$ Corumbataí, $\mathrm{DE}=\mathrm{Descalvado}, \mathrm{DO}=\mathrm{Dourado}, \mathrm{GP}=\mathrm{Gavião}$ Peixoto, $\mathrm{GU}$ = Guatapará, IB = Ibaté, IP = Ipeúna, IT = Itirapina, JB = Jaboticabal, LA = Luís Antônio, MA = Monte Alto, MT = Matão, RB = Ribeirão Bonito, RI = Rincão, SC = São Carlos, SP = São Pedro, $\mathrm{TO}=$ Torrinha, $\mathrm{TR}=$ Trabijú, $\mathrm{T}=$ occurring along all municipalities. Flowering period: JAN $=$ January, FEV = February, MAR $=$ March, ABR $=\mathrm{April}, \mathrm{MAI}=\mathrm{May}, \mathrm{JUN}=\mathrm{June}, \mathrm{JUL}=\mathrm{July}, \mathrm{AGO}$ $=$ August, $\mathrm{SET}=$ September, $\mathrm{OUT}=$ October, NOV $=$ November, $\mathrm{DEZ}=$ December

\begin{tabular}{|c|c|c|c|c|c|}
\hline Espécie & Hábito & Habitat & Município & Floração & Voucher \\
\hline Acianthera aphthosa (Lindl.) Pridgeon \& M.W.Chase & $\mathrm{E}$ & FR & $\begin{array}{l}\text { AN, AR, BR, CH, CO, DE, IP, } \\
\text { IT, JB, MT, SC, TO,TR }\end{array}$ & JUL-AGO & HUFSCAR 7282 \\
\hline Acianthera aurantiaca (Barb.Rodr.) Campacci * & $\mathrm{E}$ & FR & AN, BR, DE, IT, MT, SC & OUT-DEZ & HUFSCAR 7283 \\
\hline Acianthera bidentula (Barb.Rodr.) Pridgeon \& M.W.Chase & $\mathrm{E}, \mathrm{R}$ & FR, FES & $\mathrm{T}$ & NOV-MAI & HUFSCAR 7284 \\
\hline Acianthera bragae (Ruschi) F. Barros & $\mathrm{E}, \mathrm{R}$ & FR & $\mathrm{AN}, \mathrm{BR}, \mathrm{CH}, \mathrm{CO}, \mathrm{DE}, \mathrm{IT}, \mathrm{SC}$ & SET-NOV & HUFSCAR 7285 \\
\hline Acianthera crinita (Barb. Rodr.) Pridgeon \& M.W. Chase * & $\mathrm{E}$ & $\mathrm{FR}$ & $\begin{array}{l}\text { AN, BR, DE, DO, IT, MT, SC, } \\
\text { SP, TO }\end{array}$ & FEV-ABR & HUFSCAR 7286 \\
\hline Acianthera leptotifolia (Barb. Rodr.) Pridgeon \& M.W. Chase & $\mathrm{E}$ & FR, FES & $\mathrm{T}$ & JUN-JUL & HUFSCAR 7287 \\
\hline Acianthera macuconensis (Barb. Rodr.) F. Barros & $\mathrm{E}$ & $\mathrm{FR}$ & $\mathrm{T}$ & DEZ-MAR & HUFSCAR 7288 \\
\hline Acianthera pubescens (Lindl.) Pridgeon \& M.W. Chase & $\mathrm{E}$ & FR & $\mathrm{T}$ & DEZ-FEV & HUFSCAR 7289 \\
\hline Acianthera recurva (Lindl.) Pridgeon \& M.W. Chase & $\mathrm{E}$ & FR, FES & $\mathrm{T}$ & JAN-MAR & HUFSCAR 7290 \\
\hline Acianthera saundersiana (Rchb.f.) Pridgeon \& M.W. Chase & $\mathrm{E}$ & FR & AR, GU, LA, MT, MA & MAI-SET & HUFSCAR 7291 \\
\hline Acianthera saurocephala (Lodd.) Pridgeon \& M.W.Chase * & $\mathrm{E}$ & FR, FES & AN, BR & OUT-DEZ & HUFSCAR 7292 \\
\hline Acianthera sp. $1 *$ & $\mathrm{E}$ & FR & $\mathrm{BR}$ & AGO & HUFSCAR 7293 \\
\hline Acianthera sp. $2 *$ & $\mathrm{E}$ & FR & $\mathrm{SC}$ & OUT-NOV & HUFSCAR 7294 \\
\hline Anathallis obovata (Lindl.) Pridgeon \& M.W. Chase & $\mathrm{E}, \mathrm{R}$ & FR, FES & AN,BE,BR,CH,DE, GP,MT,SC & ABR-MAI & HUFSCAR 7295 \\
\hline
\end{tabular}




\begin{tabular}{|c|c|c|c|c|c|}
\hline Espécie & Hábito & Habitat & Município & Floração & Voucher \\
\hline Anathallis sp. & $\mathrm{E}$ & FES & $\mathrm{SC}$ & JAN-JUL & $\begin{array}{l}\text { UEC } 148369, \\
\text { HUFSCAR } 7296\end{array}$ \\
\hline Aspasia lunata Lindl. * & $\mathrm{E}$ & FR & $\mathrm{BR}$ & NOV-JAN & HUFSCAR 7297 \\
\hline Aspidogyne argentea (Vell.) Garay & $\mathrm{T}$ & FR, FES & $\mathrm{T}$ & NOV-FEV & HUFSCAR 7298 \\
\hline Aspidogyne longicornu (Cogn.) Garay & $\mathrm{T}$ & FR & $\begin{array}{l}\text { BE, CO, DE, GP, GU, IB, IT, } \\
\text { MA, MT, RI, SC }\end{array}$ & SET-OUT & HUFSCAR 7299 \\
\hline Barbosella cf. crassifolia (Edwall) Schltr. * & $\mathrm{E}$ & FR, FES & $\mathrm{DE}$ & OUT-NOV & HUFSCAR 7305 \\
\hline Baskervilla sp. * & $\mathrm{T}$ & $\mathrm{FR}$ & IT, SC & ABR-MAI & $\begin{array}{l}\text { UEC } 148371, \\
\text { HUFSCAR } 7306\end{array}$ \\
\hline Bletia catenulata Ruiz \& Pav. * & $\mathrm{T}$ & FR, CLE & $\mathrm{AR}, \mathrm{SC}$ & OUT-NOV & HUFSCAR 7307 \\
\hline Brasiliorchis chrysantha (Barb. Rodr.) R. Singer, S. Koehler \& Carnevali & $\mathrm{E}, \mathrm{R}$ & FR, FES & $\begin{array}{l}\text { AN, BR, CH, IB, IP, IT, JB, } \\
\text { MT, MA, SC, SP, TO, TR }\end{array}$ & SET-OUT & HUFSCAR 7308 \\
\hline Brasiliorchis consanguinea (Klotzsch.) R. Singer, S. Koehler \& Carnevali & $\mathrm{E}, \mathrm{R}$ & FR, FES & $\begin{array}{l}\text { AN, BR, CH, IB, IP, IT, JB, } \\
\text { MT, MA, SC, TO }\end{array}$ & JAN-FEV & HUFSCAR 7309 \\
\hline Brasiliorchis picta (Hook.) R. Singer, S. Koehler \& Carnevali & $\mathrm{E}, \mathrm{R}$ & FR & $\mathrm{AN}, \mathrm{BR}$ & DEZ-FEV & HUFSCAR 7310 \\
\hline Brassavola tuberculata Hook. * & $\mathrm{E}, \mathrm{R}$ & FR, FES & $\mathrm{AR}, \mathrm{BE}, \mathrm{BR}, \mathrm{DO}, \mathrm{MT}, \mathrm{SC}$ & JUN-JUL & HUFSCAR 7311 \\
\hline Bulbophyllum cantagallense (Barb. Rodr.) Cogn. & $\mathrm{E}$ & FR, FES & AN, BR, DE, IT & FEV-ABR & HUFSCAR 7312 \\
\hline Bulbophyllum cloroglossum Rchb.f. & $\mathrm{E}$ & CRD, FR, FES & $\mathrm{T}$ & NOV-DEZ & HUFSCAR 7313 \\
\hline Bulbophyllum dusenii Kraenzl. * & $\mathrm{E}$ & FES & SC & MAR-ABR & HUFSCAR 7314 \\
\hline Bulbophyllum aff. insetiferum Barb. Rodr. * & $\mathrm{E}$ & FES & AN & AGO & HUFSCAR 7315 \\
\hline Bulbophyllum ipanemense Hoehne & $\mathrm{E}, \mathrm{R}$ & FR, FES & $\mathrm{T}$ & ABR-MAI & HUFSCAR 7316 \\
\hline Bulbophyllum laciniatum (Barb. Rodr.) Cogn. * & $\mathrm{E}$ & FR & $\mathrm{BR}, \mathrm{IT}, \mathrm{SC}$ & SET-OUT & HUFSCAR 7317 \\
\hline Bulbophyllum plumosum (Barb. Rodr.) Cogn. * & $\mathrm{E}$ & CRD, FES & IT, SC & SET-OUT & HUFSCAR 7318 \\
\hline Bulbophyllum tripetalum Lindl. * & $\mathrm{E}$ & FR & DO & FEV-MAR & HUFSCAR 7319 \\
\hline Bulbophyllum sp.1* & $\mathrm{E}$ & FR & $\mathrm{BR}$ & - & HUFSCAR 7320 \\
\hline Bulbophyllum sp.2* & $\mathrm{E}$ & CRD, FES & $\mathrm{SC}$ & - & HUFSCAR 7321 \\
\hline Campylocentrum aromaticum Barb. Rodr. & $\mathrm{E}$ & $\mathrm{FR}$ & $\mathrm{DE}$ & JAN-FEV & HUFSCAR 7322 \\
\hline Campylocentrum brachycarpum Cogn. & $\mathrm{E}$ & FR & AN, BR, IT, SC, SP & MAR & HUFSCAR 7323 \\
\hline Campylocentrum grisebachii Cogn. & $\mathrm{E}$ & FR & $\mathrm{T}$ & MAR-ABR & HUFSCAR 7324 \\
\hline Campylocentrum micranthum (Lindl.) Rolfe & $\mathrm{E}$ & FR, FES & $\mathrm{T}$ & FEV-ABR & HUFSCAR 7325 \\
\hline
\end{tabular}




\begin{tabular}{|c|c|c|c|c|c|}
\hline Espécie & Hábito & Habitat & Município & Floração & Voucher \\
\hline Campylocentrum neglectum Cogn. & $\mathrm{E}$ & FR & AN, BR, IT, SC & JAN-MAR & HUFSCAR 7326 \\
\hline Campylocentrum pauloense Hoehne \& Schltr. & $\mathrm{E}$ & FR & AN, BR, IT, SC & JAN-MAR & HUFSCAR 7327 \\
\hline Campylocentrum rhomboglossum Hoehne \& Schltr. & $\mathrm{E}$ & FR & $\mathrm{T}$ & NOV-JAN & HUFSCAR 7328 \\
\hline Campylocentrum sellowii (Rchb.f.) Rolfe * & $\mathrm{E}$ & FR & AN & FEV-ABR & HUFSCAR 7329 \\
\hline Campylocentrum ulei Cogn. * & $\mathrm{E}$ & FR & $\mathrm{BR}$ & ABR-JUN & HUFSCAR 7330 \\
\hline Campylocentrum sp. $1 *$ & $\mathrm{E}$ & FR & AN & JAN-MAR & HUFSCAR 7331 \\
\hline Capanemia micromera Barb. Rodr. & $\mathrm{E}$ & FR & $\mathrm{T}$ & MAR-ABR & HUFSCAR 7332 \\
\hline Capanemia gehrthii Hoehne * & $\mathrm{E}$ & FR, FES & $\begin{array}{l}\text { AN, AR, BR, CO, DE, JB, } \\
\text { IT, LA, MT, SC, SP, TO }\end{array}$ & JUL-AGO & HUFSCAR 7333 \\
\hline Catasetum cernuum (Lindl.) Rchb.f. * & $\mathrm{E}$ & FR & AN, BR, DO, IT, MT, RB, SC & OUT-DEZ & HUFSCAR 7334 \\
\hline Catasetum fimbriatum (Lindl.) Paxton & $\mathrm{E}, \mathrm{R}, \mathrm{T}$ & $\begin{array}{l}\text { CR, CRD, } \\
\text { FR, FES }\end{array}$ & $\mathrm{T}$ & NOV-ABR & HUFSCAR 7335 \\
\hline Catasetum macrocarpum Rich. * & $\mathrm{E}$ & FR & $\mathrm{AR}, \mathrm{MT}$ & JAN-FEV & HUFSCAR 7336 \\
\hline Cattleya x dolosa Rchb.f. & $\mathrm{E}, \mathrm{R}$ & FR, FES & $\mathrm{AN}, \mathrm{BE}, \mathrm{DE}, \mathrm{RB}$ & ABR-JUN & HUFSCAR 7337 \\
\hline Cattleya forbesi Lindl. * & $\mathrm{E}$ & FR & $\mathrm{BR}$ & OUT-NOV & HUFSCAR 7338 \\
\hline Cattleya loddigesii Lindl. & $\mathrm{E}, \mathrm{R}$ & FR, FES & $\mathrm{T}$ & MAI-AGO & HUFSCAR 7340 \\
\hline Cattleya walkeriana Gardner & $\mathrm{E}, \mathrm{R}$ & FR, FES, CRD & $\begin{array}{l}\text { AN,AR, BE, DE, DO, GP, GU, IP } \\
\text { IT, JB, LA, MT, MA, RB, TO, TR }\end{array}$ & MAR-MAI & HUFSCAR 7341 \\
\hline $\begin{array}{l}\text { Christensonella vernicosa (Barb. Rodr.) } \\
\text { Szlach., Mytnik, Górniak \& Smiszek* }\end{array}$ & $\mathrm{E}$ & FR, FES & $\mathrm{AN}, \mathrm{BR}, \mathrm{SC}$ & NOV-JUN & HUFSCAR 7342 \\
\hline Christensonella neowiedii (Rchb.f.) S. Koehler * & $\mathrm{E}$ & FR & $\mathrm{AN}, \mathrm{BR}, \mathrm{SP}$ & NOV-FEV & HUFSCAR 7343 \\
\hline Chytroglossa paulensis Edwall * & $\mathrm{E}$ & FR & $\mathrm{BR}, \mathrm{SP}$ & OUT-NOV & HUFSCAR 7344 \\
\hline Cleistes gracilis Schltr. & $\mathrm{T}$ & FR, CLE & SC & JAN-MAR & HUFSCAR 7345 \\
\hline Cleistes metallina Schltr. & $\mathrm{T}$ & CCR, CLE & BR, IT, SC & MAR-MAI & HUFSCAR 7346 \\
\hline Corymborchis flava (Sw.) Kuntze & $\mathrm{T}$ & FR, FES & $\mathrm{T}$ & FEV-ABR & HUFSCAR 7347 \\
\hline Cranichis candida (Barb. Rodr.) Cogn. & $\mathrm{T}$ & FR & AN, AR, BR, DE, IP, IT, RI & MAI-JUN & HUFSCAR 7348 \\
\hline Cyclopogon argyrifolius (Barb.Rodr.) Barb.Rodr & $\mathrm{T}$ & CCR & $\mathrm{BR}, \mathrm{IT}, \mathrm{SC}$ & $\mathrm{ABR}$ & HUFSCAR 7349 \\
\hline Cyclopogon calophyllus Barb. Rodr. & $\mathrm{T}$ & FR, FES & $\mathrm{T}$ & AGO-SET & HUFSCAR 7350 \\
\hline Cyclopogon congestus (Vell.) Hoehne & $\mathrm{T}, \mathrm{R}$ & FES & $\mathrm{T}$ & AGO-SET & HUFSCAR 7351 \\
\hline
\end{tabular}




\begin{tabular}{|c|c|c|c|c|c|}
\hline Espécie & Hábito & Habitat & Município & Floração & Voucher \\
\hline Cyclopogon elatus (Sw.) Schltr. & $\mathrm{T}$ & FR, FES & $\mathrm{T}$ & AGO-SET & HUFSCAR 7352 \\
\hline Cyclopogon variegatus Barb. Rodr. & $\mathrm{T}$ & FR, FES & $\mathrm{T}$ & AGO-SET & HUFSCAR 7354 \\
\hline Cyrtopodium eugenii (Rchb. f. \& Warm.) Schltr. & $\mathrm{T}, \mathrm{R}$ & $\mathrm{CR}, \mathrm{CCR}$ & $\begin{array}{l}\text { AN, AR, BR, DE, DO, IT, JB, } \\
\text { MT, MA, RB, SC, TR }\end{array}$ & MAI-JUL & HUFSCAR 7355 \\
\hline Cyrtopodium gigas (Vell.) Hoehne * & $\mathrm{E}$ & $\mathrm{FR}$ & AR, BE, BR, IT, JB, MT & OUT-NOV & HUFSCAR 7356 \\
\hline Cyrtopodium hatschbachii Pabst * & $\mathrm{T}$ & FR, CLE & AR, BE, TR & AGO-OUT & HUFSCAR 7357 \\
\hline Cyrtopodium paludicolum Hoehne & $\mathrm{T}$ & FR, CLE & $\begin{array}{l}\text { AN, AR, BR, CH, CO, DE, GP, } \\
\text { IP, IT, MT, MA, RI, SC, TO }\end{array}$ & FEV-ABR & HUFSCAR 7358 \\
\hline Cyrtopodium saintlegerianum Rchb.f. * & $\mathrm{E}$ & FR & AR, MT, MA & AGO-SET & HUFSCAR 7359 \\
\hline Dryadella aviceps (Rchb.f.) Luer & $\mathrm{E}$ & FR & $\mathrm{T}$ & DEZ-ABR & HUFSCAR 7360 \\
\hline Elleanthus brasiliensis Rchb.f. * & $\mathrm{E}$ & FR & $\mathrm{AN}, \mathrm{CO}$ & DEZ-MAR & HUFSCAR 7361 \\
\hline Eltroplectris cf. triloba (Lindl.) Pabst & $\mathrm{T}$ & $\mathrm{CR}, \mathrm{CRD}$ & AN, AR, IT, DE, MT, MA, SC & SET-OUT & HUFSCAR 7362 \\
\hline Encyclia argentinensis (Speg.) Hoehne * & $\mathrm{E}$ & FR & $\mathrm{DO}, \mathrm{RI}$ & OUT-DEZ & HUFSCAR 7363 \\
\hline Encyclia flava (Lindl.) Porto \& Brade * & $\mathrm{E}, \mathrm{R}$ & CRD, FR, FES & MA & OUT-DEZ & HUFSCAR 7364 \\
\hline Encyclia oncidioides (Lindl.) Schltr. * & $\mathrm{E}$ & FR & $\mathrm{DE}$ & AGO-SET & HUFSCAR 7365 \\
\hline Encyclia patens Hook. & $\mathrm{E}, \mathrm{R}$ & FR, FES & $\mathrm{T}$ & MAI-SET & HUFSCAR 7366 \\
\hline Encyclia pauciflora (Barb. Rodr.) Porto \& Brade * & $\mathrm{E}$ & FR & $\mathrm{AN}, \mathrm{AR}, \mathrm{BR}, \mathrm{DO}, \mathrm{SC}$ & OUT-DEZ & HUFSCAR 7367 \\
\hline Epidendrum avicola Lindl. & $\mathrm{E}$ & FR, FES & $\mathrm{T}$ & JAN-FEV & HUFSCAR 7409 \\
\hline Epidendrum caldense Barb. Rodr. * & $\mathrm{E}$ & FR & SP & ABR-JUN & HUFSCAR 7368 \\
\hline Epidendrum cristatum Ruiz \& Pav. * & $\mathrm{E}$ & $\mathrm{FR}$ & IT & NOV-DEZ & HUFSCAR 7369 \\
\hline Epidendrum dendrobioides Thunb. & $\mathrm{T}$ & FR, CLE & AR, BE, BR, IT, MT, SC & DEZ-FEV & HUFSCAR 7370 \\
\hline Epidendrum densiflorum Hook. & $\mathrm{E}, \mathrm{T}, \mathrm{R}$ & FR, FES & $\mathrm{T}$ & SET-OUT & HUFSCAR 7371 \\
\hline Epidendrum denticulatum Barb. Rodr. & $\mathrm{E}, \mathrm{T}, \mathrm{R}$ & $\begin{array}{l}\text { CR, CCR, } \\
\text { FES, FR }\end{array}$ & $\mathrm{T}$ & ANOTODO & HUFSCAR 7372 \\
\hline Epidendrum difforme Jacq. & $\mathrm{E}$ & FR & $\mathrm{T}$ & ABR-JUN & HUFSCAR 7373 \\
\hline Epidendrum henschenii Barb. Rodr. * & $\mathrm{E}$ & FR & AN, TO & FEV-ABR & $\begin{array}{l}\text { UEC } 148372, \\
\text { HUFSCAR } 7374\end{array}$ \\
\hline Epidendrum latilabre Lindl. & $\mathrm{E}$ & FR & $\mathrm{T}$ & JAN-FEV & HUFSCAR 7375 \\
\hline Epidendrum martianum Lindl. * & $\mathrm{T}, \mathrm{R}$ & CCR & IT & FEV-MAR & HUFSCAR 7376 \\
\hline Epidendrum rigidum Jacq. & $\mathrm{E}$ & FR & $\mathrm{T}$ & DEZ-MAR & HUFSCAR 7377 \\
\hline
\end{tabular}




\begin{tabular}{|c|c|c|c|c|c|}
\hline Espécie & Hábito & Habitat & Município & Floração & Voucher \\
\hline Epidendrum tridactylum Lindl. & $\mathrm{E}$ & CRD, FR, FES & $\mathrm{BR}, \mathrm{IT}, \mathrm{SC}$ & DEZ-MAR & HUFSCAR 7378 \\
\hline Epistephium sclerophyllum Lindl. * & $\mathrm{T}$ & CCR & $\mathrm{AR}, \mathrm{SC}$ & FEV-ABR & HUFSCAR 7379 \\
\hline Eulophia alta (L.) Fawc. \& Rendle & $\mathrm{T}$ & CCR, FR & $\mathrm{T}$ & FEV-MAR & HUFSCAR 7380 \\
\hline Eurystyles actinosophila (Barb. Rodr.) Schltr. & $\mathrm{E}$ & FR, FES & $\begin{array}{l}\text { AN, BR, CH, DE, GP, IT, } \\
\text { MT, RI, SC, SP }\end{array}$ & FEV-ABR & HUFSCAR 7381 \\
\hline Galeandra beyrichii Rchb.f. & $\mathrm{T}, \mathrm{R}$ & CCR, FR,FES & AN, AR, DE, IT, JB, MA & JAN-MAR & HUFSCAR 7382 \\
\hline Galeandra junceoides Barb. Rodr. * & $\mathrm{T}$ & CCR & $\mathrm{AR}, \mathrm{MA}$ & FEV-ABR & HUFSCAR 7383 \\
\hline Galeandra montana Barb. Rodr. & $\mathrm{T}, \mathrm{R}$ & CCR & $\mathrm{AN}, \mathrm{AR}, \mathrm{IT}, \mathrm{SC}$ & FEV-ABR & HUFSCAR 7384 \\
\hline Galeandra styllomisantha (Vell.) Hoehne * & $\mathrm{T}$ & CCR & AR, IT & FEV-ABR & HUFSCAR 7385 \\
\hline Gomesa crispa (Lindl.) Klotzsch ex Rchb.f. * & $\mathrm{E}$ & FR & AN, IT, BR & MAR-MAI & HUFSCAR 7386 \\
\hline Gomesa blanchetii (Rchb.f.) M.W.Chase \& N.H.Williams & $\mathrm{T}, \mathrm{R}$ & CR,CCR,CLE & $\mathrm{AN}, \mathrm{IT}, \mathrm{BR}, \mathrm{SC}$ & FEV-ABR & HUFSCAR 7438 \\
\hline Gomesa echinata (Barb.Rodr.) M.W.Chase \& N.H.Williams * & $\mathrm{E}$ & FR & $\mathrm{BR}$ & SET-NOV & HUFSCAR 7301 \\
\hline Gomesa fimbriata (Lindl.) A.C. Ferreira, M.I.S. Lima \& Pansarin & $\mathrm{E}$ & FR & AN, BR, IT, SC, SP, TO & OUT-NOV & HUFSCAR 7300 \\
\hline Gomesa hydrophila (Barb.Rodr.) M.W.Chase \& N.H.Williams * & $\mathrm{T}, \mathrm{E}$ & $\begin{array}{l}\text { FR, CLECRD, } \\
\text { FR, FES }\end{array}$ & AN, IT, BR, SC & OUT-DEZ & HUFSCAR 7439 \\
\hline Gomesa praetexta (Rchb.f.) M.W.Chase \& N.H.Williams * & $\mathrm{E}$ & FR, FES & $\mathrm{BR}$ & FEV-ABR & HUFSCAR 7440 \\
\hline Gomesa pubes (Lindl.) M.W.Chase \& N.H.Williams & $\mathrm{E}$ & FR & AN, BR, IT, SC, SP, TO & AGO-OUT & HUFSCAR 7303 \\
\hline Gomesa radicans (Rchb.f.) M.W.Chase \& N.H.Williams * & $\mathrm{E}$ & $\mathrm{FR}$ & $\mathrm{BR}$ & FEV-MAR & HUFSCAR 7444 \\
\hline Gomesa sarcodes (Lindl.) M.W.Chase \& N.H.Williams * & $\mathrm{E}$ & FR & $\mathrm{AN}, \mathrm{BR}$ & NOV-DEZ & HUFSCAR 7304 \\
\hline Gomesa varicosa (Lindl.) M.W.Chase \& N.H.Williams & & & AN, BE, GP, GU, LA, SC & FEV-MAI & HUFSCAR 7441 \\
\hline Gomesa venusta (Drapiez) M.W.Chase \& N.H.Williams * & $\mathrm{E}$ & FR & $\mathrm{BR}, \mathrm{SP}$ & NOV-JAN & HUFSCAR 7442 \\
\hline Gongora bufonia Lindl. * & $\mathrm{E}$ & FR & $\mathrm{BR}$ & NOV-DEZ & HUFSCAR 7387 \\
\hline Govenia utriculata (Sw.) Lindl. & $\mathrm{T}$ & FR, CLE & $\begin{array}{l}\text { AN, AR, BE, BR, CH, IT, } \\
\text { MT, RI, SC, TO }\end{array}$ & NOV-JAN & HUFSCAR 7388 \\
\hline Grobya amherstiae Lindl. * & $\mathrm{E}$ & FR & BR, TO & FEV-MAR & HUFSCAR 7389 \\
\hline Habenaria fastor Warm. ex Hoehne & $\mathrm{T}$ & CLE,FR & AR, IT, SC & JAN-FEV & HUFSCAR 7390 \\
\hline Habenaria glazioviana Kraenzl. ex Cogn. & $\mathrm{T}$ & CLE,FR & $\mathrm{T}$ & FEV-MAR & HUFSCAR 7391 \\
\hline Habenaria hamata Barb. Rodr. & $\mathrm{T}$ & CR,CLE & IT & MAR-MAI & HUFSCAR 7392 \\
\hline Habenaria johannensis Barb. Rodr. & $\mathrm{T}$ & CLE & BE, IT & JAN-MAR & HUFSCAR 7393 \\
\hline
\end{tabular}




\begin{tabular}{|c|c|c|c|c|c|}
\hline Espécie & Hábito & Habitat & Município & Floração & Voucher \\
\hline Habenaria aff. josephensis Barb. Rodr. & $\mathrm{T}$ & $\mathrm{FR}$ & IP, SC & FEV-ABR & HUFSCAR 7394 \\
\hline Habenaria obtusa Lindl. & $\mathrm{T}$ & CR,CCR,CLE & $\mathrm{IT}, \mathrm{SC}$ & FEV-ABR & HUFSCAR 7395 \\
\hline Habenaria parviflora Lindl. & $\mathrm{T}$ & CLE & $\mathrm{BR}, \mathrm{IT}$ & DEZ-JAN & HUFSCAR 7396 \\
\hline Habenaria petalodes Lindl. & $\mathrm{T}$ & $\mathrm{FR}$ & IT, SC & MAR-MAI & HUFSCAR 7397 \\
\hline Habenaria repens Nutt. * & $\mathrm{T}$ & CCR & $\mathrm{BR}, \mathrm{IT}$ & FEV-MAR & HUFSCAR 7398 \\
\hline Habenaria rodeiensis Barb. Rodr. * & $\mathrm{T}$ & CLE & JB, MA, MT & $\mathrm{FEV}$ & HUFSCAR 7399 \\
\hline Habenaria schwackei Barb.Rodr. * & $\mathrm{T}$ & CLE & $\mathrm{JB}, \mathrm{MA}$ & JAN & HUFSCAR 7400 \\
\hline Habenaria trifida Kunth * & $\mathrm{T}$ & CCR & JB, MT & DEZ-JAN & HUFSCAR 7401 \\
\hline Hapalorchis lineatus (Lindl.) Schltr. * & $\mathrm{T}$ & FR, FES & $\mathrm{AN}, \mathrm{BR}, \mathrm{SP}$ & AGO & HUFSCAR 7402 \\
\hline Heterotaxis valenzuelana (A. Rich.) Ojeda \& Carnevali * & $\mathrm{E}, \mathrm{R}$ & FR & AN, BR, DE, IT, SC & FEV-ABR & HUFSCAR 7403 \\
\hline Houlettia brocklehurstiana Lindl. & $\mathrm{T}$ & FR & $\mathrm{T}$ & FEV-ABR & HUFSCAR 7404 \\
\hline Ionopsis utricularioides (Sw.) Lindl. & $\mathrm{E}$ & $\begin{array}{l}\text { CR, CRD, } \\
\text { FR, FES }\end{array}$ & $\mathrm{T}$ & AGO-SET & HUFSCAR 7405 \\
\hline Isabelia violacea (Lindl.) C. van den Berg \& M.W. Chase & $\mathrm{E}, \mathrm{R}$ & FR, FES & $\mathrm{T}$ & AGO-SET & HUFSCAR 7406 \\
\hline Isabelia virginalis Barb. Rodr. & $\mathrm{E}$ & FR, FES & $\mathrm{T}$ & MAI-JUN & HUFSCAR 7407 \\
\hline Isochilus linearis (Jacq.) R.Br. & $\mathrm{E}$ & FR, FES & $\mathrm{T}$ & DEZ-MAI & HUFSCAR 7408 \\
\hline Lankesterella caespitosa (Lindl.) Hoehne & $\mathrm{E}$ & FR, FES & $\mathrm{T}$ & JAN-FEV & HUFSCAR 7410 \\
\hline Leptotes unicolor Barb. Rodr. * & $\mathrm{E}$ & FR & $\mathrm{BR}$ & JUN-JUL & HUFSCAR 7411 \\
\hline Ligeophila stigmatoptera (Rchb.f.) Garay & $\mathrm{T}$ & FR, FES & $\begin{array}{l}\mathrm{AN}, \mathrm{AR}, \mathrm{BR}, \mathrm{CH}, \mathrm{CO}, \\
\mathrm{DE}, \mathrm{SC}, \mathrm{TO}\end{array}$ & NOV-MAR & HUFSCAR 7412 \\
\hline Liparis nervosa (Thunb.) Lindl. & $\mathrm{T}$ & FR, FES & $\mathrm{T}$ & DEZ-JAN & HUFSCAR 7413 \\
\hline Liparis sp. & $\mathrm{T}$ & FR & BE, IT & JAN-FEV & HUFSCAR 7414 \\
\hline Lockhartia lunifera (Lindl.) Rchb.f. * & $\mathrm{E}$ & FR & TO & DEZ-FEV & HUFSCAR 7415 \\
\hline Macradenia multiflora (Kraenzl.) Cogn.* & $\mathrm{E}$ & FR & $\mathrm{BR}$ & JAN-ABR & HUFSCAR 7416 \\
\hline Malaxis cf. jaraguae (Hoehne \& Schltr.) Pabst & $\mathrm{T}$ & FR & $\mathrm{SC}$ & JAN-MAR & HUFSCAR 7417 \\
\hline Malaxis sp. * & $\mathrm{T}$ & FR & $\mathrm{SC}$ & JAN-FEV & HUFSCAR 7418 \\
\hline Mesadenella cuspidata (Lindl.) Garay & $\mathrm{T}$ & FR, FES & $\mathrm{T}$ & JAN-MAI & HUFSCAR 7419 \\
\hline Microcattleya cattleyoides (Rich.) V.P. Castro \& Chiron * & $\mathrm{E}$ & $\mathrm{FR}$ & $\mathrm{BR}$ & JUN-JUL & HUFSCAR 7420 \\
\hline Microchilus arietinus (Rchb.f. \& Warm.) Ormed & $\mathrm{T}$ & FR & $\mathrm{T}$ & OUT-NOV & HUFSCAR 7421 \\
\hline Microlaelia lundii (Rchb.f. \& Warm.) Chiron \& V.P. Castro & $\mathrm{E}$ & FR & $\mathrm{T}$ & MAI-AGO & HUFSCAR 7422 \\
\hline
\end{tabular}




\begin{tabular}{|c|c|c|c|c|c|}
\hline Espécie & Hábito & Habitat & Município & Floração & Voucher \\
\hline Miltonia flavescens Lindl. & $\mathrm{E}, \mathrm{R}$ & FR, FES & $\mathrm{T}$ & OUT-NOV & HUFSCAR 7423 \\
\hline Miltonia regnellii Rchb.f. & $\mathrm{E}$ & FR, FES & MT & JAN-ABR & HUFSCAR 7424 \\
\hline Mormodes sinuata Rchb.f. \& Warm. * & $\mathrm{E}$ & FR & MA, MT & FEV-MAR & HUFSCAR 7425 \\
\hline Mormolyca rufescens (Lindl.) M. A. Blanco \& Carnevali * & $\mathrm{E}$ & FR, FES & $\mathrm{T}$ & JAN-FEV & HUFSCAR 7426 \\
\hline Myoxanthus lonchophyllus (Barb. Rodr.) Luer & $\mathrm{E}$ & FR & $\mathrm{T}$ & JUL-MAR & HUFSCAR 7427 \\
\hline Myoxanthus pulvinatus (Barb. Rodr.) Luer* & $\mathrm{E}$ & FR & SP & DEZ-MAR & HUFSCAR 7428 \\
\hline Notylia cf. hemitricha Barb. Rodr. & $\mathrm{E}$ & $\mathrm{FR}$ & AN, BR, IT, DE, SC & AGO-SET & HUFSCAR 7429 \\
\hline Notylia cf. lyrata S. Moore & $\mathrm{E}$ & FR & $\begin{array}{l}\text { AN, AR, BR, CH, CO, DE, } \\
\text { DO, IT, SC, SP, TO }\end{array}$ & AGO-SET & HUFSCAR 7431 \\
\hline Notylia nemorosa Barb. Rodr. & $\mathrm{E}$ & FR & $\mathrm{AN}, \mathrm{BR}$ & JUL-AGO & HUFSCAR 7432 \\
\hline Octomeria aloifolia Barb. Rodr. * & $\mathrm{E}$ & FR, FES & $\mathrm{BR}, \mathrm{IT}, \mathrm{SC}$ & OUT-DEZ & HUFSCAR 7433 \\
\hline Octomeria cf. alpina Barb. Rodr. * & $\mathrm{E}$ & FR, FES & $\mathrm{AN}$ & MAI-JUN & HUFSCAR 7434 \\
\hline Octomeria pinicola Barb. Rodr. & $\mathrm{E}, \mathrm{R}$ & FR, FES & $\mathrm{T}$ & JUN-JUL & HUFSCAR 7435 \\
\hline Octomeria sp. * & $\mathrm{E}$ & FR & $\mathrm{SC}$ & - & HUFSCAR 7436 \\
\hline Oeceoclades maculata (Lindl.) Lindl. & $\mathrm{T}$ & $\begin{array}{l}\text { CR, CCR, } \\
\text { CRD, FR, FES }\end{array}$ & $\mathrm{T}$ & JAN-ABR & HUFSCAR 7437 \\
\hline Ornithocephalus myrticola Lindl. * & $\mathrm{E}$ & FR & $\begin{array}{l}\text { AN, AR, BR, DE, IT, MT, } \\
\text { MA, SC, SP }\end{array}$ & NOV-JAN & HUFSCAR 7443 \\
\hline Ornitophora radicans (Rchb.f.) Garay \& Pabst & $\mathrm{E}$ & $\mathrm{FR}$ & $\mathrm{BR}$ & FEV-MAR & HUFSCAR 7353 \\
\hline Pabstiella tripterantha (Rchb.f.) F. Barros & $\mathrm{E}$ & FR & $\mathrm{T}$ & JUN-NOV & HUFSCAR 7445 \\
\hline Pelexia bonariensis (Lindl.) Schltr. & $\mathrm{T}$ & $\mathrm{CR}, \mathrm{CCR}$ & $\mathrm{BR}, \mathrm{IT}$ & MAI-JUN & HUFSCAR 7446 \\
\hline Pelexia aff. itatiaye Schltr. & $\mathrm{T}$ & $\mathrm{FR}$ & AN, BR, DE, DO, RB, SC & AGO-SET & HUFSCAR 7447 \\
\hline Pelexia laminata Schltr. & $\mathrm{T}$ & $\mathrm{CR}, \mathrm{CCR}$ & $\mathrm{BR}, \mathrm{IT}$ & JAN-MAR & HUFSCAR 7448 \\
\hline Pelexia laxa (Poepp. \& Endl.) Lindl. & $\mathrm{T}$ & FES & DO & SET-NOV & HUFSCAR 7449 \\
\hline Pelexia oestrifera (Rchb.f. \& Warm.) Schltr. & $\mathrm{T}$ & $\mathrm{CR}, \mathrm{CCR}$ & MT & JUL-AGO & HUFSCAR 7450 \\
\hline Pelexia sp.1 & $\mathrm{T}$ & FR & IT & FEV-MAR & $\begin{array}{l}\text { UEC } 148368, \\
\text { HUFSCAR } 7451\end{array}$ \\
\hline Pelexia sp. $2 *$ & $\mathrm{~T}$ & $\mathrm{CR}$ & $\mathrm{SC}$ & SET & HUFSCAR 7452 \\
\hline Phragmipedium vittatum (Vell.) Rolfe * & $\mathrm{T}, \mathrm{R}$ & FR, CLE & BE, AR, DE, GP, IT, SC & DEZ-ABR & HUFSCAR 7453 \\
\hline Phymatidium delicatulum Lindl. * & $\mathrm{E}$ & FR & $\mathrm{BR}, \mathrm{SP}, \mathrm{TO}$ & MAR-ABR & HUFSCAR 7454 \\
\hline
\end{tabular}




\begin{tabular}{|c|c|c|c|c|c|}
\hline Espécie & Hábito & Habitat & Município & Floração & Voucher \\
\hline Pleurobotryum atropurpureum Barb. Rodr. * & $\mathrm{E}$ & FR & IT, SC & ABR-JUN & HUFSCAR 7455 \\
\hline Polystachya concreta (Jacq.) Garay \& H.R. Sweet* & $\mathrm{E}$ & FR, FES & BR & JAN-FEV & HUFSCAR 7457 \\
\hline Polystachya estrellensis Rchb.f. & $\mathrm{E}, \mathrm{R}$ & FR, FES & $\mathrm{T}$ & JAN-FEV & HUFSCAR 7458 \\
\hline Polystachya foliosa (Hook.) Rchb.f. & $\mathrm{E}$ & FR, FES & $\mathrm{T}$ & JAN-FEV & HUFSCAR 7459 \\
\hline Prescottia oligantha (Sw.) Lindl. * & $\mathrm{T}$ & FR & $\mathrm{BR}, \mathrm{SC}$ & AGO-SET & HUFSCAR 7460 \\
\hline Prescottia stachyodes (Sw.) Lindl. & $\mathrm{T}$ & FR & $\mathrm{DE}, \mathrm{SC}$ & AGO-OUT & HUFSCAR 7461 \\
\hline Prescottia sp. * & $\mathrm{T}$ & CCR & IT & AGO-SET & HUFSCAR 7462 \\
\hline Prosthechea calamaria Lindl. * & $\mathrm{E}$ & FES & $\mathrm{AN}, \mathrm{SC}$ & MAI-JUN & HUFSCAR 7463 \\
\hline Prosthechea glumacea Lindl. * & $\mathrm{E}, \mathrm{R}$ & FES & AN & JAN-FEV & HUFSCAR 7464 \\
\hline Pseudomaxillaria parviflora (Poepp. \& Endl.) Garay* & $\mathrm{E}$ & FR, FES & $\mathrm{AN}, \mathrm{BR}$ & FEV-MAR & HUFSCAR 7465 \\
\hline Psilochilus modestus Barb. Rodr. & $\mathrm{T}$ & FR & $\mathrm{T}$ & DEZ-MAR & HUFSCAR 7466 \\
\hline Pteroglossa roseoalba (Rchb.f.) Salazar \& M.W. Chase & $\mathrm{T}$ & FR, FES & $\begin{array}{l}\text { AN, BR, DE, DO, IB, IT, } \\
\text { MT, MA, SC }\end{array}$ & ABR-MAI & HUFSCAR 7467 \\
\hline Rodriguezia decora (Lem.) Rchb.f. & $\mathrm{E}, \mathrm{T}$ & $\begin{array}{l}\text { CR, CRD, } \\
\text { FR, FES }\end{array}$ & $\mathrm{T}$ & MAR-JUN & HUFSCAR 7468 \\
\hline Rodriguezia obtusifolia (Lindl.) Rchb.f. * & $\mathrm{E}$ & FR & MT, SC & MAR-ABR & HUFSCAR 7469 \\
\hline Sacoila lanceolata (Aubl.) Garay & $\mathrm{T}$ & $\begin{array}{l}\text { CR, CCR, } \\
\text { CLE,CRD,FES }\end{array}$ & $\mathrm{S}^{\mathrm{T}}$ & SET-OUT & HUFSCAR 7470 \\
\hline Sanderella discolor (Barb. Rodr.) Cogn. * & $\mathrm{E}$ & FR & $\begin{array}{l}\text { AN, BR, CH, CO, DE, IT, } \\
\text { MT, SC, SP, TO }\end{array}$ & ABR-MAI & HUFSCAR 7471 \\
\hline Saundersia mirabilis Rchb.f. * & $\mathrm{E}$ & FR & SP, TO & OUT-NOV & HUFSCAR 7472 \\
\hline Sobralia decora Bateman * & $\mathrm{T}$ & FR & $\mathrm{BR}$ & MAR & HUFSCAR 7473 \\
\hline Sarcoglottis cf. schwackei (Cogn.) Schltr. * & $\mathrm{T}$ & CLE,FR & SC & SET-OUT & HUFSCAR 7474 \\
\hline Sarcoglottis fasciculata (Vell.) Schltr. * & $\mathrm{T}$ & FES & $\mathrm{AN}, \mathrm{BR}, \mathrm{IT}, \mathrm{MT}$ & AGO-SET & HUFSCAR 7475 \\
\hline Sarcoglottis ventricosa (Vell.) Hoehne * & $\mathrm{T}$ & FES & AN, BR, IT & AGO-SET & HUFSCAR 7476 \\
\hline Sauroglossum nitidum (Vell.) Schltr. & $\mathrm{T}$ & FR & $\mathrm{T}$ & JUL-OUT & HUFSCAR 7477 \\
\hline Scaphyglottis modesta (Rchb.f.) Schltr. * & $\mathrm{E}$ & FR & $\mathrm{AN}, \mathrm{BR}, \mathrm{MT}, \mathrm{JB}, \mathrm{SC}$ & MAR-ABR & HUFSCAR 7478 \\
\hline Schomburgkia gloriosa Rchb.f. & $\mathrm{E}$ & CRD, FR, FES & $S \mathrm{~T}$ & MAI-JUN & HUFSCAR 7479 \\
\hline Sophronitis cernua Lindl. & $\mathrm{E}, \mathrm{R}$ & CRD, FR, FES & $\mathrm{S} \mathrm{T}$ & FEV-ABR & HUFSCAR 7480 \\
\hline Specklinia aristulata (Lindl.) Luer & $\mathrm{E}$ & FR & AN, AR, BE, BR, DO, DE, SC & MAR-ABR & HUFSCAR 7481 \\
\hline
\end{tabular}




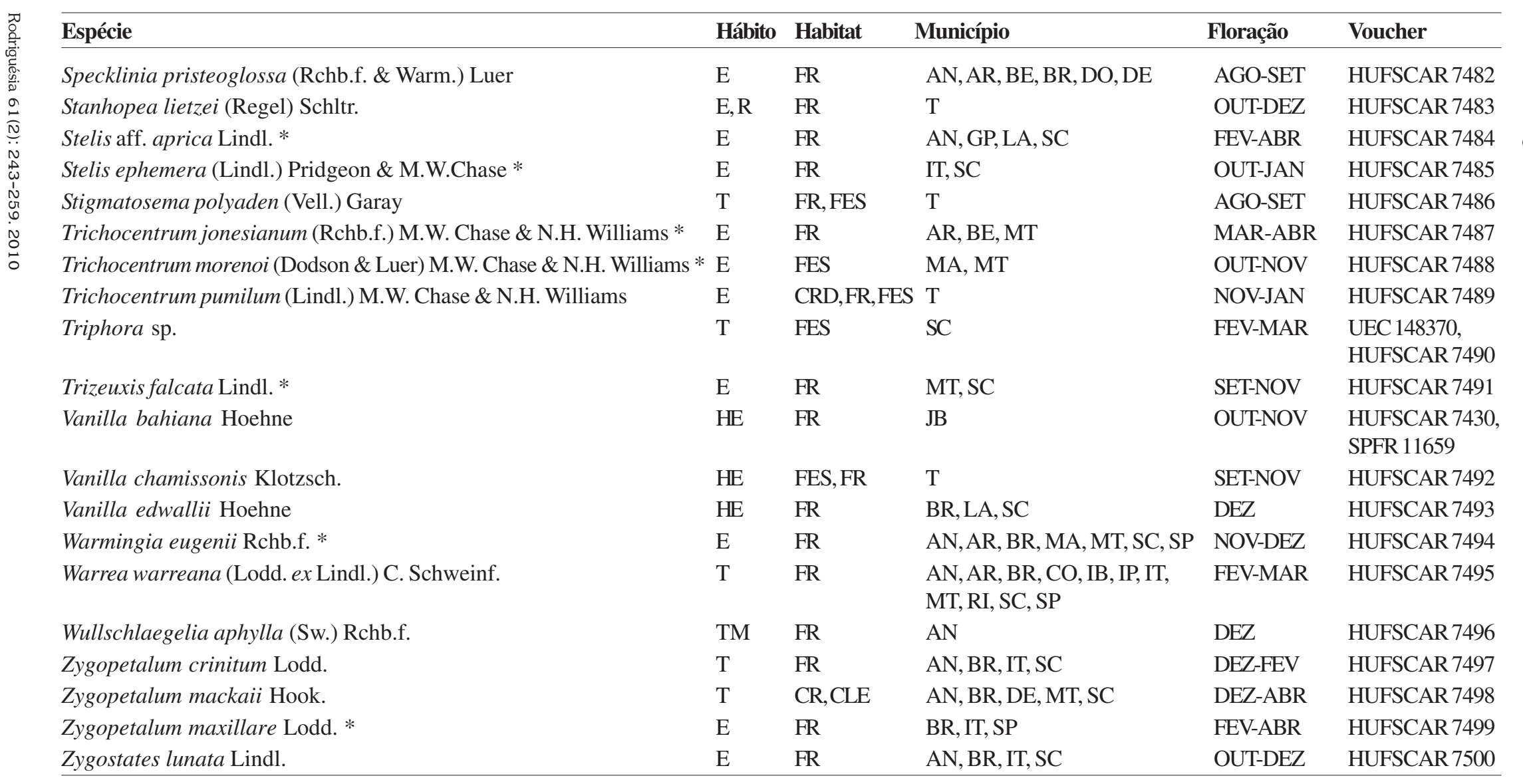

* Espécie rara na região de estudo

Material depositado nos herbários HUEFSCAR e UEC = coletor A.W.C. Ferreira

Material depositado no herbário SPFR = coletor E.R. Pansarin 
No presente levantamento de espécies de Orchidaceae, notável foi a ausência de espécies dos gêneros Bifrenaria Lindl., geralmente encontrada em áreas de floresta atlântica ombrófila densa (Barros 1983; Miller et al. 1996), Cerrado e floresta mesófila estacional semidecidual (MeniniNeto et al. 2007; Pansarin \& Pansarin 2008), e também de Trigonidium Lindl., que ocorre em regiões de floresta atlântica ombrófila densa (Romanini \& Barros 2007), de Cerrado (Batista \& Bianchetti 2003) e de floresta amazônica (Ribeiro et al. 1999).

Embora Scuticaria itirapinensis Pabst tenha sido descrita com base em um espécime coletado na porção central do estado de São Paulo, em uma região próxima ao município de Itirapina (Pabst 1973), ela não foi encontrada no decorrer das expedições de campo efetuadas no presente levantamento, parecendo estar extinta localmente. Segundo histórico de alguns mateiros, essa espécie ocorria na região do Morro do Baú e na Serra de Itaqueri, em Itirapina, tendo sido coletada até seu extermínio populacional, ainda na década de 1980.

Cleistes gracilis Schltr., Cleistes metallina Schltr., Psilochilus modestus Barb. Rodr. e Triphora sp., apresentam picos de floração distintos, todos eles com as flores abrindo sincronicamente. A tendência de muitas flores abrirem ao mesmo tempo pode estar relacionada à otimização da ação dos polinizadores, um fenômeno que tem sido documentado para outras Triphoreae, como Triphora trianthophora (Sw.) Rydb. (Williams 1994) e Psilochilus modestus Barb. Rodr. (Pansarin \& Amaral 2008).

O fato da maioria das espécies $(64 \%)$ de orquídeas na região central de São Paulo ocorrerem como epífitas (140 spp.), contrastando com os $35 \%$ de terrícolas (77 spp.), pode estar relacionado com a predominância das fisionomias florestais (florestas mesófilas, matas galeria e cerradões) nessa região ecotonal. Em ambientes de cerrado sensu stricto existe a predominância de espécies terrícolas de Orchidaceae, ficando as espécies epífitas concentradas principalmente em matas de galeria (Batista \& Bianchetti 2003; Batista et al. 2005).

De acordo com Dressler (1981, 1993), em média, duas em cada três espécies de Orchidaceae são epífitas. Devido à dependência da umidade e do substrato arbóreo, a diversidade de epífitas pode ser usada como indicador ecológico de qualidade e conservação de florestas úmidas. Por exigir adaptações específicas, o epifitismo pode proporcionar maior acesso à luz e diminuição da competição (Kersten 2006). As espécies epífitas correspondem à parte significativa da diversidade vegetal e contribuem positivamente para tornar as florestas tropicais úmidas um dos mais complexos ecossistemas da Biosfera (Kersten 2006). Segundo Nadkarni (1986), a capacidade destas florestas em abrigarem grande número de animais pode ser atribuída ao substrato e sustento providos pelas epífitas, e por sua respectiva capacidade de retenção de nutrientes da chuva, neblina e partículas em suspensão. Os ambientes úmidos, próximos ao mar e em desnível costumam ser as áreas mais ricas em espécies de Orchidaceae, como ocorre na Serra do Mar, no Brasil, e nas montanhas da Colômbia e do Equador (Pabst \& Dungs 1975; Dressler 1993). Nossos dados para a região central de São Paulo sustentam os argumentos sobre a tendência dos ambientes mais úmidos serem favoráveis ao estabelecimento de espécies de Orchidaceae. De fato, a floresta ripícola foi a que apresentou o maior número de espécies (89\%), seguido pela floresta mesofítica estacional semidecidual (36\%), pelo campo cerrado $(9,6 \%)$, pelo campo limpo $(9,1 \%)$ e pelo cerrado e cerradão $(8,2 \%)$. Mesmo nos ambientes dentro do Cerrado, existe uma tendência da maioria das espécies de Orchidaceae estarem concentradas em campos úmidos e em florestas de galeria (Batista \& Bianchetti 2003; Batista et al. 2005).

A maioria das espécies de Orchidaceae da região central de São Paulo $(63,4 \%)$ floresce no outono, seguidas pelas que florescem no verão $(62,1 \%)$ e pelas que florescem na primavera $(47 \%)$ (Tab. 1). Esses resultados são semelhantes aos encontrados por Pansarin \& Pansarin (2008) para as Orchidaceae da Serra do Japi, no interior do estado de São Paulo. O fato da maior parte das espécies florescerem na época mais chuvosa e quente (primavera, verão e parte do outono), coincide com ser esta a época mais favorável para o metabolismo da maioria das espécies vegetais (Marschner 1995; Nardoto et al.2006), é também aquela em que a maioria dos insetos polinizadores costuma estar ativo (muitas espécies podem ser encontradas apenas nesse período) ou apresentar maior número populacional (e.g., Agostini \& Sazima 2003).

Das espécies encontradas nos locais de estudo durante a realização do trabalho, 44,7\% são raras. $\mathrm{O}$ fato de que nessa área há 15 espécies de Orchidaceae em comum com a Lista Oficial das Plantas Ameaçadas de Extinção do estado de São Paulo (SMA 2004), reforça que esta área deve ser prioritária para conservação e preservação.

Alguns fatores naturais, como a dinâmica da sucessão ecológica parecem estar contribuindo para o declínio populacional de algumas orquídeas 
da região. Populações de Cleistes gracilis e Houlettia brocklehurstiana que ocorrem em fragmentos florestais do município de São Carlos, têm sido afetadas pelo sombreamento resultante do avanço da mata nativa adjacente à área palustre (campo), onde ambas as espécies de orquídeas ocorrem. Inicialmente, a paisagem era dominada por briófitas (principalmente Sphagnum sp.), por touceiras de Lagenocarpus sp. (Cyperaceae), e pequenas árvores e arbustos esparsos (principalmente espécies de Myrtaceae). Desde o início das coletas (1996) até o momento, observou-se que árvores começaram a colonizar essa área, sombreando e alterando o regime hídrico, afetando as populações das referidas orquídeas, que atualmente são encontradas apenas em pequenas clareiras, em áreas mais iluminadas.

Além dos fatores naturais, o desmatamento para a expansão da lavoura canavieira contribuiu muito para a redução das áreas de vegetação nativa ocupada por populações de Orchidaceae na região central de São Paulo. Nos poucos fragmentos que restaram, as coletas indiscriminadas feitas por mateiros e orquidófilos da região têm sido responsáveis pelos percentuais de espécies ameaçadas aqui determinado. Muitas populações de Cattleya loddigesii e Cattleya walkeriana foram praticamente dizimadas por ação de coletores de orquídeas. De forma semelhante, populações de Catasetum fimbriatum têm sido prejudicadas pela derrubada de macaúbas (Acrocomia aculeata (Jacq.) Lodd. ex Mart. Arecaceae), especialmente na região entre Itirapina e Brotas (A.W.C. Ferreira, observações pessoais). Assim como a porção central do estado de São Paulo, outras áreas ecotonais nativas de São Paulo também vêm sofrendo com as interferências antrópicas (Pansarin \& Pansarin 2008).

Caso medidas fiscalizadoras e preventivas (como a educação ambiental escolar e das populações que têm acesso a essas áreas) não forem tomadas, estimase que muitas dessas espécies de orquídeas, sobretudo as ornamentais, poderão ser extintas localmente, em processo semelhante ao que ocorre com diversas espécies de Orchidaceae e outras famílias de angiospermas que ocorrem em regiões da Floresta Atlântica sensu lato, reduzida a 5-8\% de sua formação original (Dean 1995; Morellato \& Haddad 2000).

\section{Agradecimentos}

Ao tecnólogo Carlos Aparecido Casali, do Departamento de Botânica da Universidade Federal de São Carlos (UFSCar) e ao Programa de PósGraduação em Ecologia e Recursos Naturais da
UFSCar o auxílio nas idas ao campo; aos orquidófilos Anderson Hideki Shitara, José Luis Teixeira, Osvaldo Roberto Rigon, João Carlos Martins, João Carlos, Aparecido Alves, Alcides Medeiros e Rodrigo Pierobon o auxílio nas idas ao campo; ao ilustrador botânico Ricardo Milanetti Degani e ao Prof. Dr. Marcos Arduin (Laboratório de Anatomia Vegetal - UFSCar) o auxílio com as imagens; aos responsáveis pelo projeto Orchidstudium, Dalton Holland Baptista e Américo Docha Neto o apoio nas pesquisas bibliográficas; aos inúmeros proprietários e ao IBAMA (processo número 02001.003951/2006-50) e Instituto Florestal de São Paulo (processo número 40.380/2006) a permissão de acesso às propriedades e autorização para pesquisa e, à CAPES a bolsa de doutoramento concedida ao primeiro autor.

\section{Referências}

Agostini, K. \& Sazima, M. 2003. Plantas ornamentais e seus recursos para abelhas no campus da Universidade Estadual de Campinas, estado de São Paulo, Brasil. Áreas Básicas/Botânica. Bragantia 62: 335-343.

Atzinger, N.V.; Cardoso, A.L.R. \& Ilkiu-Borges, A.L. 1996. Flora orquidológica da Serra das Andorinhas, São Geraldo do Araguaia - PA. Boletim do Museu Paraense Emílio Goeldi, série Botânica 12: 59-74.

Barros, F. 1983. Flora fanerogâmica da Reserva do Parque Estadual das Fontes do Ipiranga (São Paulo, Brasil). 198 - Orchidaceae. Hoehnea 10: 74-124.

Batista, J.A.N. \& Bianchetti, L.B. 2003. Lista atualizada das Orchidaceae do Distrito Federal. Acta Botanica Brasilica 17: 183-201.

Batista, J.A.N.; Bianchetti, L.B. \& Pellizzaro, K.F. 2005. Orchidaceae da Reserva Biológica do Guará, DF, Brasil. Acta Botanica Brasilica 19: 221-232.

Braga, P.I.S. 1977. Aspectos biológicos das Orchidaceae de uma campina da Amazônia Central. Acta Amazonica (suplemento) 7: 1-89.

Cardoso, J.C. \& Israel, M. 2005. Levantamento de espécies da família Orchidaceae em Águas de Santa Bárbara (SP) e seu cultivo. Horticultura Brasileira 23: 169-173.

Chase, M.W.; Cameron, K.M.; Barrett, R.L. \& Freudenstein, J.V. 2003. DNA data and Orchidaceae systematics: a new phylogenetic classification. In: Dixon, K.W.; Kell, S.P.; Barrett, R.L. \& Cribb, P.J. (eds.). Orchid conservation. Natural History Publications, Kota Kinabalu, Sabah. Pp. 69-89.

Chase, M.W.; Williams, N.H.; Faria, A.D.; Neubig, K.M.; Amaral, M.C.E. \& Whitten, W.M. 2009. Floral convergence in Oncidiinae (Cymbidieae; Orchidaceae): an expanded concept of Gomesa and a new genus Nohawilliamsia. Annals of Botany 104: 387-402. 
Cogniaux, A. 1893-1896. Orchidaceae. In: Martius, C.F.P.; Eichler, A.G. \& Urban, I. (ed.). Flora brasiliensis. F. Fleischer, Munique. Vol. 3. Pp. 1-672.

Cogniaux, A. 1898-1902. Orchidaceae. In: Martius, C.F.P.; Eichler, A.G. \& Urban, I. (ed.). Flora brasiliensis. R. Oldenbourg, Munique. Vol. 3. Pp. 1-664.

Cogniaux, A. 1904-1906. Orchidaceae. In: Martius, C.F.P.; Eichler, A.G. \& Urban, I. (ed.). Flora brasiliensis. R. Oldenbourg, Munique. Vol. 3. Pp. 1-604.

Coutinho L.M. 1978. O conceito de Cerrado. Revista Brasileira de Botânica 1: 17-23.

Dean, W. 1995. A ferro e fogo: a história e a devastação da Mata Atlântica brasileira. Cia. das Letras, São Paulo. 484p.

Dressler, R.L. 1981. The orchids: natural history and classification. Havard University Press, Cambridge. 332p.

Dressler, R.L. 1993. Phylogeny and classification of the orchid family. Portland, Dioscorides Press. 314p.

Dressler, R.L. 2005. How many orchid species? Selbyana 26: $155-158$.

Durigan, G.; Baitello, J. B.; Franco, G.A.D. C. \& Siqueira, M.F. 2004. Plantas do cerrado paulista: imagens de uma paisagem ameaçada. Ed. Páginas \& Letras, São Paulo. 475p.

Garay, L.A. 1977. Systematics of the Physurinae (Orchidaceae) in the new world. Bradea 2: 191-204.

Hoehne, F.C. 1940. Orchidaceas. In: Hoehne, F.C. (ed.). Flora brasilica. Vol. 12. Secretaria da Agricultura do Estado de São Paulo, São Paulo. Pp. 1-254.

Hoehne, F.C. 1942. Orchidaceas. In: Hoehne, F.C. (ed.). Flora brasilica. Vol. 12. Secretaria da Agricultura do Estado de São Paulo, São Paulo. Pp. 1-218.

Hoehne, F.C. 1945 Orchidaceas. In: Hoehne, F.C. (ed.). Flora brasilica. Vol. 12. Secretaria da Agricultura do Estado de São Paulo, São Paulo. Pp. 1-389.

Hoehne, F.C. 1949. Iconografia de orquidáceas do Brasil. S.A. Indústrias "Graphicars-f. Lanzara", São Paulo. $601 \mathrm{p}$.

Hoehne, F.C. 1953. Orchidaceas. In: Hoehne, F.C. (ed.). Flora brasilica. Vol. 12. Secretaria da Agricultura do Estado de São Paulo, São Paulo. Pp. 1-397.

Kersten, R.A. 2006. Epifitismo vascular na bacia do alto Iguaçu, Paraná. Curitiba. Tese de Doutorado. Universidade Federal do Paraná, Curitiba. 218p.

Köppen, W.P. 1948. Climatologia: con un estudio de los climas de la tierra. Fondo de Cultura Econômica, México. 478p.

Kronka, F.J.N.; Matsukuma, C.K.; Nalon, M.A.; Cali, I.H.D.; Rossi, M.; Mattos, I.F.A.; Shin-Ike, M.S. \& Pontinhas, A.A.S. 1993. Inventário florestal do estado de São Paulo. Instituto Florestal de São Paulo, São Paulo. 199p.

Kronka, F.J.N.; Nalon, M.A.; Matsukuma, C.K.; Pavão, M.; Guillaumon, J.R.; Cavalli, A.C.; Giannotti, E.; Iwane, M.S.S.; Lima, L.M.P.R.; Montes, J.; Del
Cali, I.H. \& Haack, P.G. 1998. Áreas do domínio do cerrado no estado de São Paulo. Secretaria de Estado do Meio Ambiente, Instituto Florestal, São Paulo. 84p.

Luer, C.A. 1986a. Icones Pleurothallidinarum I. Systematics of Pleurothallidinae (Orchidaceae). Monographs in Systematic Botany from the Missouri Botanical Garden 15: 1-81.

Luer, C.A. 1986b. Icones Pleurothallidinarum II. Systematics of Pleurothallidinae (Orchidaceae). Monographs in Systematic Botany from the Missouri Botanical Garden 16: 1-63.

Luer, C.A. 1986c. Icones Pleurothallidinarum III. Systematics of Pleurothallidinae (Orchidaceae). Monographs in Systematic Botany from the Missouri Botanical Garden 20: 1-109.

Marschner, H. 1995. Mineral nutrition of higher plants. Academic Press, San Diego. 889p.

Menini Neto, L.; Alves, R.J.V.; Barros, F. \& Forzza, R.C. 2007. Orchidaceae do Parque Estadual de Ibitipoca, MG, Brasil. Acta Botanica Brasilica 21: 687-696.

Miller, D.; Warren, R. \& Miller, I.M. 1996. Orquídeas do alto da serra da mata atlântica pluvial do sudeste do Brasil. Lis Gráfica e Editora, Rio de Janeiro. 256p.

Morellato, L.P.C. \& Haddad, C.F.B. 2000. The Brazilian Atlantic Forest: an introduction. Biotropica 32: 786-792.

Myers, N.; Mittermeier, R.A.; Mittermeier, C.G.; Fonseca, G.A.B. \& Kent, J. 2000. Biodiversity hotspots for conservation priorities. Nature 403: 853-858.

Nadkarni, N.M. 1986. An ecological overview and checklist of vascular epiphytes in the Monteverde cloud forest reserve, Costa Rica. Brenesia 24: 55-632.

Nardoto, G.B.; Bustamante, M.M.C.; Pinto, A.S. \& Klink, C.A. 2006. Nutrient use efficiency at ecosystem and species level in savanna areas of Brazil and impacts of fire. Journal of Tropical Ecology 22: 191-201.

Pabst, G.F.J. 1950. Notas sôbre "Polystachya estrellensis, Rchb. f.". Orquidea 12: 167-169.

Pabst, G.F.J. 1973. Additamenta ad Orchidologiam Brasiliensem - XIV. Bradea 1: 327-344.

Pabst, G.F.J. \& Dungs, F. 1975. Orchidaceae brasilienses. Vol. 1. Kurt Schmersow, Hildesheim. 408p.

Pabst, G.F.J. \& Dungs, F. 1977. Orchidaceae brasilienses. Vol. 2. Kurt Schmersow, Hildesheim. 418p.

Pansarin, E.R. \& Amaral, M.C.E. 2008. Pollen and nectar as a reward in the basal epidendroid Psilochilus modestus (Orchidaceae: Triphoreae): A study of floral morphology, reproductive biology and pollination strategy. Flora 203: 474-483.

Pansarin, E.R. \& Pansarin, L.M. 2008. A família Orchidaceae na Serra do Japi, São Paulo, Brasil. Rodriguesia 59: 99-111. 
Ramos, V.S.; Durigan, G.; Franco, G.A.D.C.; Siqueira, M.F. \& Rodrigues, R.R. 2007. Árvores da Floresta Estacional Semidecidual: guia de identificação. Instituto Florestal, Série Registros 31: 137-141.

Ribeiro, J.E.L.S. 1992. Florística e padrões de distribuição da família Orchidaceae na planície litorânea do núcleo de desenvolvimento Picinguaba, município de Ubatuba, Parque Estadual da Serra do Mar, SP. Dissertação de Mestrado. Universidade Estadual Paulista, Rio Claro. 304p.

Ribeiro, J.E.L.S.; Hopkins, M.J.G.;Vicentini, A.; Sothers, C.A.; Costa, M.A.S.; Brito, J.M.; Souza, M.A.D.; Martins, L.H.P.; Lohmann, L.G.; Assunção, P.A.C.L.; Pereira, E.C.; Silva, C.F.; Mesquita, M.R. \& Procópio, L.C. 1999. Flora da Reserva Ducke: guia de identificação das plantas vasculares de uma floresta de terra firme na Amazônia Central. Manaus, Instituto Nacional de Pesquisas da Amazônia. 816p.

Romanini, R.P. \& Barros, F. 2007. Orchidaceae. In: Melo, M.M.R.F.; Barros, F.; Chiea, S.A.C.; Kirizawa, M.; Jung-Mendaçolli, S.L. \& Wanderley, M.G.L. (eds.). Flora fanerogâmica da Ilha do Cardoso. Vol. 12. Secretaria de Estado do Meio Ambiente, São Paulo. Pp. 29-275.

Silveira, E.C.; Cardoso, A.L.R.; Ilkiu-Borges, A.L. \& Atzingen, N.V. 1995. Flora orquidológica da Serra dos Carajás, estado do Pará. Boletim do Museu Paraense Emílio Goeldi, Série Botânica 11: 75-87.

SMA - Secretaria do Meio Ambiente do Estado de São Paulo. 2004. Lista de espécies da flora ameaçadas de extinção no estado de São Paulo. Resolução SMA48, de 21.9.2004. Disponível em <http://www.ibot. sp.gov.br/resolucao_sma48/resolucao48.htm>. Acesso em 10 março 2009.

Soares, J.J.; Silva, D.W. \& Lima, M.I.S. 2003. Current state and projection of the probable original vegetation of the São Carlos region of São Paulo State, Brazil. Brazilian Journal of Biology 63: 527-536.

Sprunger, S.; Cribb, P. \& Toscano de Brito, A.L.V. 1996. João Barbosa Rodrigues - Iconographie des orchidées du Brésil. Vol. 1. The illustrations. Friedrich Reinhardt, Basle. 324p.

Toscano de Brito, A.L.V. \& Cribb, P. 2005. Orquídeas da Chapada Diamantina. Nova Fronteira, Rio de Janeiro. 400p.

Williams, S.A. 1994. Observations on reproduction in Triphora trianthophora (Orchidaceae). Rhodora 96: 30-43.

Word Checklist of Selected Plant Families. 2006. The Board of the Royal Botanic Gardens, Kew. Disponível em $<$ http://apps.kew.org/wcsp/home.do $>$. Acesso em março 2009. 